\begin{tabular}{|c|l|}
\hline Title & Storage shear modulus of columnar structure formed in an immiscible polymer blend under electric fields \\
\hline Author(s) & Aida, Kohei; Na, Y ang Ho; Nagaya, Tomoy uki; Orihara, Hiroshi \\
\hline Citation & $\begin{array}{l}\text { Physical Review E, 80(4), O41807 } \\
\text { https://doi.org/40.1103/PhysRevE.80.041807 }\end{array}$ \\
\hline Issue Date & 2009-10 \\
\hline Doc URL & http://hdl.handle.net/2115/39845 \\
\hline Rights & ○ 2009 The A American Physical Society \\
\hline Type & article \\
\hline File Information & PRE80-4_041807.pdf \\
\hline
\end{tabular}

Instructions for use 


\title{
Storage shear modulus of columnar structure formed in an immiscible polymer blend under electric fields
}

\author{
Kohei Aida, ${ }^{1}$ Yang Ho Na, ${ }^{1}$ Tomoyuki Nagaya, ${ }^{2}$ and Hiroshi Orihara,* \\ ${ }^{1}$ Department of Applied Physics, Graduate School of Engineering, Hokkaido University, Sapporo 060-8628, Japan \\ ${ }^{2}$ Department of Electrical and Electronic Engineering, Faculty of Engineering, Oita University, Oita 870-1192, Japan
}

(Received 27 March 2009; published 26 October 2009)

\begin{abstract}
Oscillatory measurements of a columnar structure were performed, which was formed in an immiscible polymer blend subjected to an electric field. The formation process was observed through a confocal scanning laser microscope and the structure for the measurements was confirmed to be well defined. The storage shear modulus at low frequencies increased with increasing the electric field. A linear relation was found between the storage shear modulus and the square of the electric field. The static modulus calculated on the basis of the Maxwell stress exerted on the interface and interfacial tension was in good agreement with the experimental result.
\end{abstract}

DOI: 10.1103/PhysRevE.80.041807

PACS number(s): $83.80 . \mathrm{Tc}, 82.70 . \mathrm{Kj}, 83.10 . \mathrm{Tv}$

\section{INTRODUCTION}

In rheology, immiscible polymer blends exhibit many interesting behaviors both under shear flow and electric fields, which are intimately related to changes in morphology [1-9]. In a typical blend of two polymers, droplets of one polymer are dispersed in the matrix of the other polymer under shear flow. In general, the polymer with higher viscosity and smaller volume fraction tends to become droplets under shear flow. When subjected to an electric field, the droplets become elongated along the field due to the Maxwell stress exerted on the interface between the two polymers, which originates in the mismatch of electrical properties such as permittivity and conductivity between them [1-9]. The elongated droplets may play a role to increase the apparent viscosity, which is called electrorheological (ER) effect. With increasing the electric field they change into complicated forms such as network [8] or stripe structure [9] through coalescence and breakup resulting in the further increase in ER effect.

In the morphological changes under shear flow and electric fields, Maxwell's stress and interfacial tension should play a crucial role. However, it is difficult to quantitatively investigate them in the complicated structures. Therefore, we turn our attention to a quiescent columnar structure formed along the electric field without shear flow. This structure has a restoring force that depends on the Maxwell stress and interfacial tension, when it is subjected to a shear strain, as will be shown later. This indicates that a quantitative investigation of them may be performed through oscillation measurements of the columnar structure.

Columnar structures have been constructed in different ways so far [10-14]. One interesting way is to use the instability of a liquid/liquid interface subjected to an electric field perpendicular to the interface; fluctuations with a characteristic wavelength grow to lead the formation of well-defined columnar structures [12-14]. Meanwhile, the simplest way is

\footnotetext{
*Author to whom correspondence should be addressed. FAX: +81-11-706-6639; orihara@eng.hokudai.ac.jp
}

to apply an electric field to a droplet-dispersed phase. The droplets are elongated along the electric field to form bridges between two electrodes, where it should be noted that certain conditions are necessary for the elongation of droplets in electrical properties [15]. Ha and Yang made dynamic frequency sweep measurements by imposing a small-amplitude oscillatory shear to a columnar structure thus obtained under electric fields [7]. However, the columnar structure was not perfect according to a photo presented in their paper; columns were not straight and broken and, moreover, many small droplets were dispersed. They have shown that the storage modulus at low frequencies increases as the applied electric field is increased, but there was no quantitative analysis.

We adopted the latter method and constructed perfect columnar structures suitable for performing oscillation measurements. We selected a blend suitable for the formation of columns, which has large Maxwell stress and small interfacial tension. The structures were observed with a confocal scanning laser microscope (CSLM) and three-dimensional (3D) images were constructed to confirm the quality of the columns and, furthermore, to measure the radius and the density of columns, which are necessary for theoretical analyses. In this paper we present the dynamic modulus of an ideal columnar structure under electric fields and compare the results with a theory developed by taking into account the Maxwell stress and interfacial tension.

\section{EXPERIMENT}

In the experiment, we used a liquid crystalline polymer (LCP) [16] (Fig. 1) and a polyisobutylene (PIB). All the experiments were done at $25^{\circ} \mathrm{C}$, where the LCP was in the isotropic phase. The mesogens play a role in enhancing the permittivity and conductivity. The viscosities, relative dielectric constants, and conductivities of LCP and PIB are, respectively, 47.0 $\mathrm{Pa} \mathrm{s}$, 30.5 $\mathrm{Pa} \mathrm{s}, 15,2.8,8.1 \times 10^{-9} \Omega^{-1} \mathrm{~m}^{-1}$, and $3.3 \times 10^{-11} \Omega^{-1} \mathrm{~m}^{-1}$ at $25^{\circ} \mathrm{C}$. We used PIB because it is a good refractive index-matching fluid with LCP; the indices of LCP and PIB are 1.486 and 1.485 , respectively. To observe the structure clearly and to distinguish LCP and PIB, 


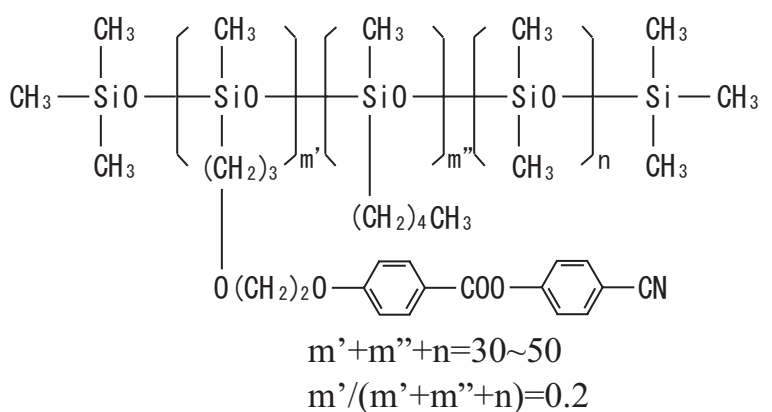

FIG. 1. Chemical structure of LCP.

a small amount of fluorescent dye, IANBD amide (Molecular Probes), was doped into LCP before mixing LCP and PIB. It was confirmed that the dye did not dissolve in PIB. They were mixed in the weight ratio of $L C P: P I B=1: 6$. In this blend, LCP was dispersed in PIB as droplets in the absence of an electric field.

A schematic picture of our system is shown in Fig. 2. The sample was sandwiched between a bottom glass plate with an indium tin oxide (ITO)-coated electrode and the rotating plate of a rheometer (MCR301, Anton Paar). The diameter of rotating plate was $20 \mathrm{~mm}$ and the gap between the plates is $200 \mu \mathrm{m}$. The observation was made through the bottom glass plate with a CSLM (IX71, OLYMPUS and CSU22, YOKOGAWA). We carried out both structural observation and rheological measurement at the same time. Electric fields were applied to the blends by a synthesizer (Multifunction Synthesizer 1940, NF Electric Instruments) and a high voltage amplifier (MODEL 609C-6, Trek). In order to construct $3 \mathrm{D}$ images, we used a piezoactuator (P-721.10, PI) with which the objective lens could move vertically. We applied a triangular voltage of $0.6 \mathrm{~Hz}$ to the piezoactuator so that the objective lens or the focal plane of the microscope periodically moved up and down between the glass surface and $75 \mu \mathrm{m}$ above it. The frame rate was $60 \mathrm{fps}$ so that 50 images were captured per half period, from which one 3D image was constructed.

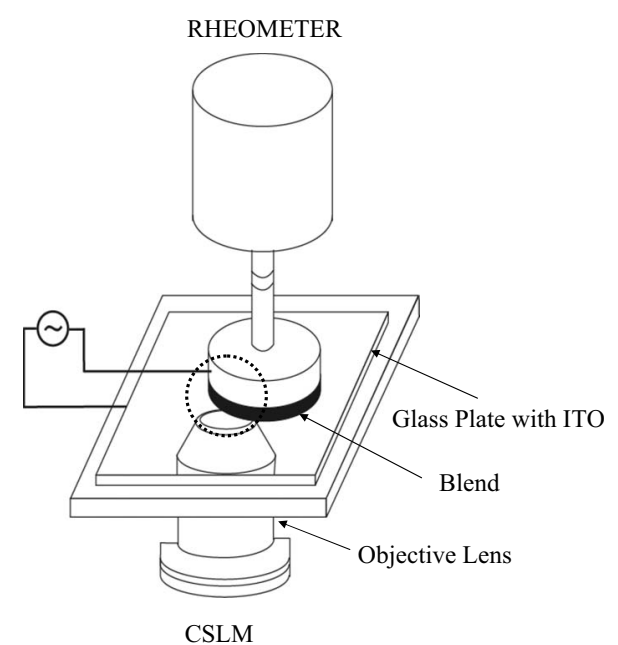

FIG. 2. Schematic of the system combining a CSLM and a rheometer.

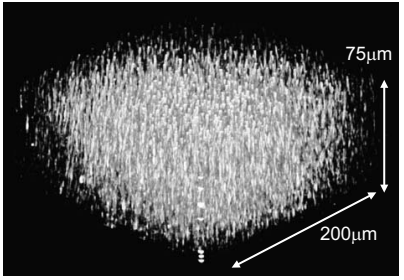

(a)

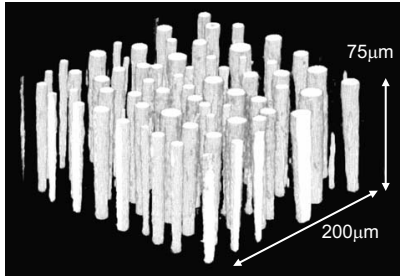

(b)
FIG. 3. Formation of columns after applying an ac electric field with an amplitude of $5 \mathrm{kV} / \mathrm{mm}$ and a frequency of $2 \mathrm{~Hz}$. Images were taken (a) $1 \mathrm{~s}$ later and (b) $100 \mathrm{~s}$ later.

\section{RESULTS AND DISCUSSION}

In this blend, LCP was dispersed in PIB as droplets in the absence of an electric field. The average droplet size is inversely proportional to the shear rate. In our experiment, first we applied a preshear to the blend to make small droplets, stopped the shear flow, and then applied an electric field. When subjected to the electric field, LCP droplets were elongated in the direction of the electric field by the electric stress exerted on the interface between LCP and PIB due to the mismatch in electric properties such as permittivity and conductivity between them and many columns were formed. The formation of columns after applying the field is shown in Fig. 3, where a preshear of $200 \mathrm{~s}^{-1}$ was applied for $1 \mathrm{~min}$ and then an ac electric field with an amplitude $5 \mathrm{kV} / \mathrm{mm}$ and a frequency $2 \mathrm{~Hz}$ was applied. It is seen that the columns coalesce and the average size increases following the formation of columns. The coarsening process will be reported in a separate paper.

Unlike the droplet-dispersed structure, in the present columnar structure the bridges of LCP were formed between the top and bottom plates so that the storage modulus was expected to increase at low frequencies. Therefore, we measured the dynamic modulus of the columnar structure. In order to keep the columnar structure, successive application of the electric field was necessary and the modulus depended on the amplitude of ac electric field. At each oscillatory measurement, the same procedure mentioned above was repeated for constructing the columnar structure and then we waited $15 \mathrm{~min}$ before the measurement so that the coalescence of columns almost ceased. We applied a small oscillating strain with an amplitude of 5\% so that the columnar structure could be maintained. It took about 3 min for each oscillation measurement, during which the structure was observed and it was confirmed that the structure did not change. To apply voltages to the sample we used a thin wire touching the rotating shaft of the rheometer. However, the friction between them made the measurement difficult at frequencies lower than $1 \mathrm{rad} / \mathrm{s}$. Figure 4 shows the frequency dispersions of the complex modulus measured at several amplitudes of ac electric field, $E_{0}$, with a frequency of $2 \mathrm{~Hz}$, including the modulus in the droplet-dispersed structure without electric field, which was prepared by applying a shear flow of $200 \mathrm{~s}^{-1}$ for $1 \mathrm{~min}$. In the droplet-dispersed state, the storage modulus is small at low angular frequencies, while at high frequencies it increases due to the elastic deformation of droplets [17]. On the other hand, in the columnar structure 


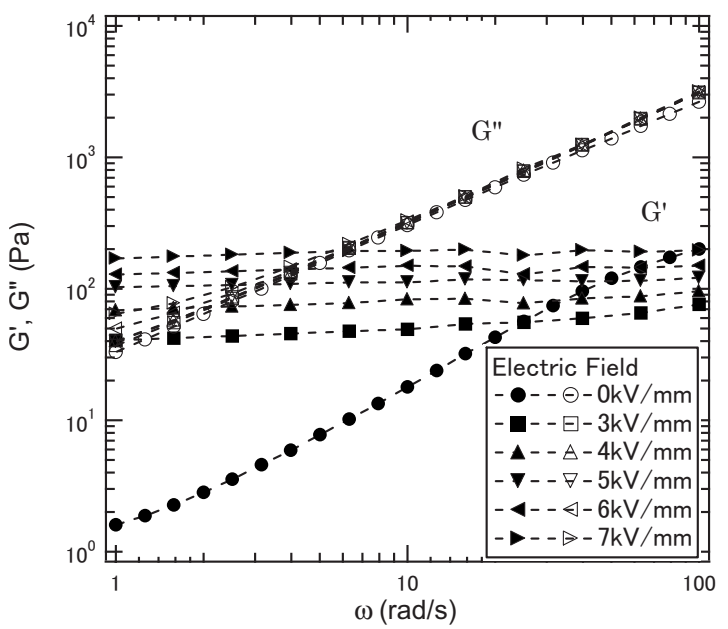

FIG. 4. Frequency dispersions of the storage modulus $\left[G^{\prime}\right.$ (closed)] and the loss modulus $\left[G^{\prime \prime}\right.$ (open)] measured at several amplitudes of ac electric field with a frequency of $2 \mathrm{~Hz}$. The oscillation amplitude is $5 \%$.

the storage modulus takes a large value at low frequencies and increases with increasing the electric field. On the other hand, the loss modula are almost the same irrespective of the electric field.

The above elasticity appearing in the columnar structure may originate in the interfacial tension between LCP and PIB and the electric stress at the interface. The interfacial energy is proportional to the surface area of columns. Therefore, when the columns tilt, the surface area, i.e., the surface energy increases resulting in a restoring force. The electric stress comes from the mismatch in electric properties such as the permittivity and conductivity between LCP and PIB, as has been mentioned before. In our blend, LCP is larger than PIB both in permittivity and conductivity. The electric stress is expressed in terms of the Maxwell stress tensor, which is proportional to the square of the electric field. This indicates that the storage modulus related to the electric stress should be proportional to the square of the applied electric field. This is illustrated in Fig. 5, where $G^{\prime}$ at $\omega=10 \mathrm{~s}^{-1}$ is plotted against $E_{0}^{2}$ at 2 and $512 \mathrm{~Hz}$. There seems to be a linear relation between $G^{\prime}$ and $E_{0}^{2}$. The value of $G^{\prime}$ extrapolated at $E_{0}=0$ is the contribution of the interfacial tension. The slope decreases as the frequency of ac electric field is increased. This frequency dependence is caused by the conductivity as will be shown.

Let us theoretically derive the static elastic modulus $G^{\prime}(0)$. The contribution of the interfacial tension $\Gamma$ to it can be calculated as follows. When a column with a radius $a$ and a height $d$ is tilted by a shear strain $\gamma$, as shown in Fig. 6(a), the interfacial area becomes $S=2 \operatorname{\pi ad}\left(1+\gamma^{2} / 4\right)$ for a small strain. Therefore, the force acting on the upper plate is $F_{t}=$ $-\partial(S \Gamma) / \partial(d \gamma)=-\pi a \Gamma \gamma$, and so $G_{t}^{\prime}(0)=\pi n a \Gamma$, where $n$ is the area density of columns. The calculation of the electrostatic contribution is more tedious. We should solve the Laplace equation for the electrostatic potential $\phi$,

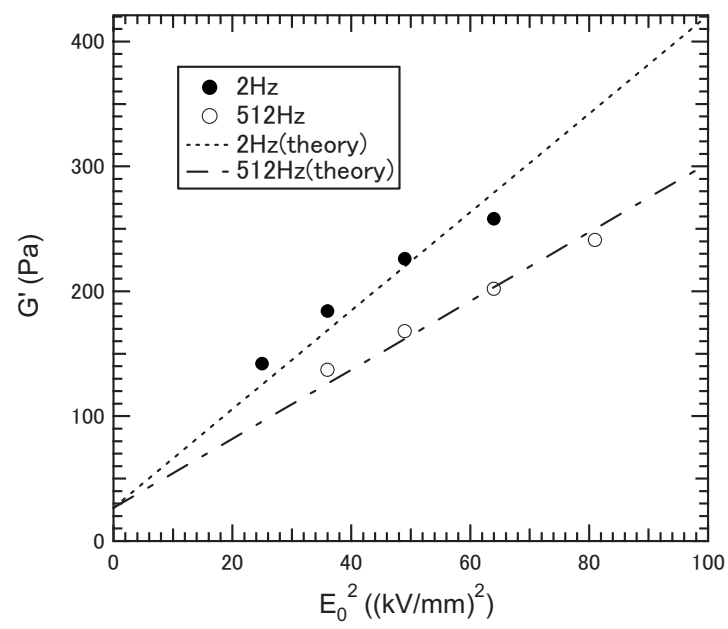

FIG. 5. Plot of the storage modulus at $\omega=10 \mathrm{~s}^{-1}$ vs the square of the amplitude of ac electric field.

$$
\Delta \phi=0,
$$

under the boundary condition at the interface,

$$
[\phi]=0,
$$

$$
[\widetilde{\varepsilon} \nabla \boldsymbol{\phi} \cdot \boldsymbol{n}]=0
$$

with

$$
\widetilde{\varepsilon}=\varepsilon+\frac{\sigma}{i \omega_{e}},
$$

where $\boldsymbol{n}$ is the unit vector normal to the interface, and $\varepsilon, \sigma$, and $\omega_{e}$ are the dielectric constant, the conductivity, and the angular frequency of the applied ac electric field (not to be confused with that of shear strain), respectively, and $[A]$ indicates the difference of the value of $A$ inside and outside the interface. In addition, we put $\phi(z=0)=0$ and $\phi(z=d)=\phi_{0}$. Here, we introduce a new coordinate system of $x^{\prime}-y^{\prime}-z^{\prime}$, in which the slant column is transformed to the vertical one, as shown in Fig. 6(b). The coordinates of $x-y-z$ and $x^{\prime}-y^{\prime}-z^{\prime}$ are related as

$$
x=x^{\prime}+y z^{\prime},
$$
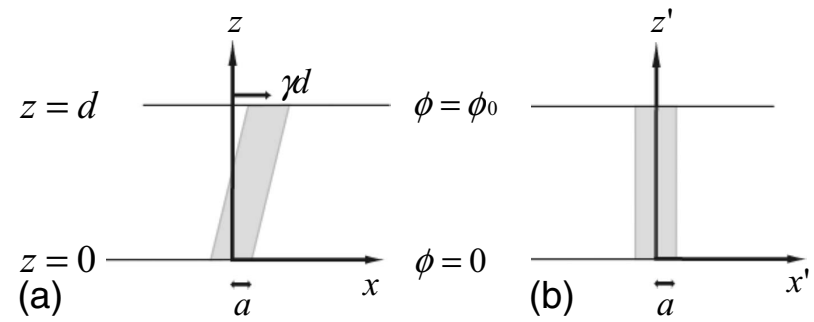

FIG. 6. (a) Schematic of one column. (b) A new coordinate system of $x^{\prime}-y^{\prime}-z^{\prime}$ in which the slant column is transformed to the vertical one. 


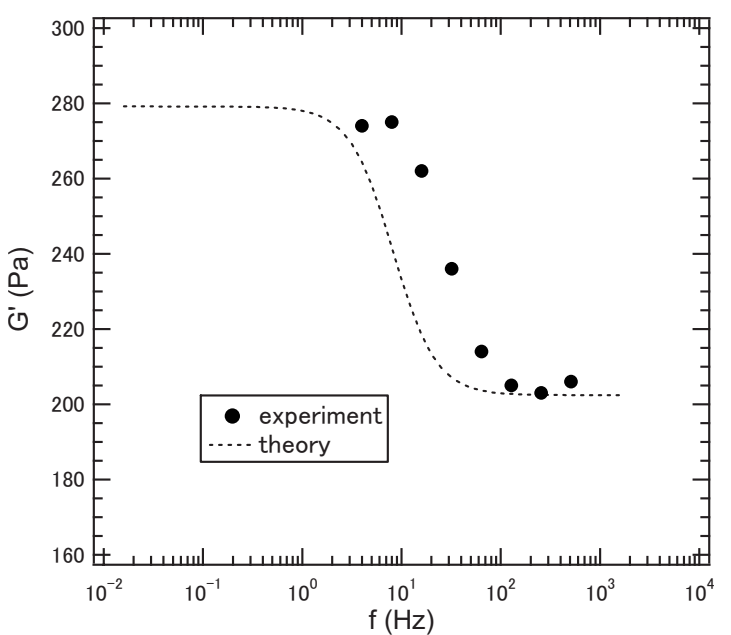

FIG. 7. Dependence of the storage modulus at $\omega=10 \mathrm{~s}^{-1}$ on the frequency of ac electric field.

$$
\begin{aligned}
& y=y^{\prime}, \\
& z=z^{\prime} .
\end{aligned}
$$

In the $x^{\prime}-y^{\prime}-z^{\prime}$ coordinate system, Eqs. (1) and (2) become

$$
\begin{gathered}
\Delta^{\prime} \phi-2 \gamma \frac{\partial^{2} \phi}{\partial x^{\prime} \partial z^{\prime}}+\gamma^{2} \frac{\partial^{2} \phi}{\partial x^{\prime 2}}=0 \\
{[\phi]=0} \\
{\left[\widetilde{\varepsilon}\left\{\nabla^{\prime} \phi \cdot \boldsymbol{n}^{\prime}-\gamma\left(\frac{\partial \phi}{\partial z^{\prime}} n_{x}^{\prime}+\frac{\partial \phi}{\partial x^{\prime}} n_{z}^{\prime}\right)+\gamma^{2} \frac{\partial \phi}{\partial x^{\prime}} n_{x}^{\prime}\right\}\right]=0 .}
\end{gathered}
$$

The above equations can be solved by the perturbation method. We expand $\phi$ with respect to $\gamma$ :

$$
\phi=\phi^{(0)}+\gamma \phi^{(1)}+\gamma^{2} \phi^{(2)}+\cdots .
$$

After some calculations, we obtain the solution in the cylindrical coordinate,

$$
x^{\prime}=\rho \cos \theta, \quad y^{\prime}=\rho \sin \theta,
$$

as

$$
\phi_{1}^{(0)}=\phi_{2}^{(0)}=\phi_{0} / d z^{\prime}
$$

$$
\begin{aligned}
& \phi_{1}^{(1)}=\sum_{m=1}^{\infty} c_{1 m} I_{1}(m \pi \rho / d) \cos \theta \sin m \pi z^{\prime} / d, \\
& \phi_{2}^{(1)}=\sum_{m=1}^{\infty} c_{2 m} K_{1}(m \pi \rho / d) \cos \theta \sin m \pi z^{\prime} / d,
\end{aligned}
$$

with

$$
\begin{aligned}
& c_{1 m}=\frac{2\left[1-(-1)^{m}\right] \phi_{0}\left(\widetilde{\varepsilon}_{1}-\widetilde{\varepsilon}_{2}\right) /\left[(m \pi)^{2} I_{1}(m \pi a / d)\right]}{\widetilde{\varepsilon}_{1} I_{1}^{\prime}(m \pi a / d) / I_{1}(m \pi a / d)-\widetilde{\varepsilon}_{2} K_{1}^{\prime}(m \pi a / d) / K_{1}(m \pi a / d)}, \\
& c_{2 m}=\frac{2\left[1-(-1)^{m}\right] \phi_{0}\left(\widetilde{\varepsilon}_{1}-\widetilde{\varepsilon}_{2}\right) /\left[(m \pi)^{2} K_{1}(m \pi a / d)\right]}{\widetilde{\varepsilon}_{1} I_{1}^{\prime}(m \pi a / d) / I_{1}(m \pi a / d)-\widetilde{\varepsilon}_{2} K_{1}^{\prime}(m \pi a / d) / K_{1}(m \pi a / d)},
\end{aligned}
$$

where subscripts " 1 " and " 2 " refer to the inside and outside of the column, respectively, and $I_{1}(x)$ and $K_{1}(x)$ are, respectively, the modified Bessel functions of the first and second kinds, and $I_{1}^{\prime}(x)$ and $K_{1}^{\prime}(x)$ are their derivatives.

The force acting on the upper plate can be expressed in terms of Maxwell's stress tensor, $T_{i j}=\varepsilon\left(E_{i} E_{j}-E^{2} / 2 \cdot \delta_{i j}\right)$, as

$$
F_{e}=\frac{1}{d} \int \sum_{j} T_{1 j} n_{j} z d S
$$

where the integration is taken all over the surface of the slant column. The right-hand side of the above equation is rewritten up to the first order of $\gamma$ as

$F_{e}=-\frac{1}{2} \gamma\left(\varepsilon_{1}-\varepsilon_{2}\right) \phi_{0} \operatorname{Re}\left\{\int \phi_{1}^{(1)} \cos \theta d S^{\prime}\left[1+\exp \left(i 2 \omega_{e} t\right)\right]\right\}$,

where the integration is taken all over the surface of the vertical column. It should be noted that the force consists of $\mathrm{dc}$ and $2 \omega_{e}$ components. In our experiments we could observe almost no deformation of columns with the frequency $2 \omega_{e} \geq 4 \pi \mathrm{rad} / \mathrm{s}$, which may be much higher than the eigenfrequencies of deformation modes in the column. Thus, we have obtained the elastic modulus owing to the electrostatic interaction. The total static elastic modulus is, therefore,

$$
G^{\prime}(0)=\pi n a \Gamma+2 n a d\left(\varepsilon_{1}-\varepsilon_{2}\right) E_{0}^{2} \operatorname{Re}\left[\sum_{m=1}^{\infty} \frac{\left[1-(-1)^{m}\right]\left(\widetilde{\varepsilon}_{1}-\widetilde{\varepsilon}_{2}\right) / m^{3} \pi^{2}}{\widetilde{\varepsilon}_{1} I_{1}^{\prime}(m \pi a / d) / I_{1}(m \pi a / d)-\widetilde{\varepsilon}_{2} K_{1}^{\prime}(m \pi a / d) / K_{1}(m \pi a / d)}\right],
$$

where $E_{0}=\phi_{0} / d$ is the amplitude of the applied ac electric field. Here, we have omitted the oscillating term coming from the $2 \omega_{e}$ term in Eq. (13), which may play no role in oscillatory measurements. It is readily understood that the electrostatic contribution disappears for the column and the matrix being the same in permittivity, that is, $\varepsilon_{1}=\varepsilon_{2}$, and it depends on the frequency of the ac electric field through the conductivity. 
From Eq. (14) we can numerically calculate the static modulus at a given ac electric field. We used the following values: $\Gamma=3 \times 10^{-3} \mathrm{Nm}^{-1}, a=8.6 \times 10^{-6} \mathrm{~m}, d=2 \times 10^{-4} \mathrm{~m}$, $n=3.3 \times 10^{8} \mathrm{~m}^{-2}, \quad \varepsilon_{1}=15 \varepsilon_{0} \mathrm{Fm}^{-1} \quad\left(\varepsilon_{0}\right.$ : permittivity of vacuum), $\varepsilon_{2}=2.8 \varepsilon_{0} \mathrm{Fm}^{-1}, \sigma_{1}=8.1 \times 10^{-9} \Omega^{-1} \mathrm{~m}^{-1}$, and $\sigma_{2}$ $=3.3 \times 10^{-11} \Omega^{-1} \mathrm{~m}^{-1}$. In Fig. 5, the calculated results are shown in a dotted line $(2 \mathrm{~Hz})$ and a dashed line $(512 \mathrm{~Hz})$, which are in good agreement with the experimental data. Next, we investigate the frequency dependence in detail. Figure 7 shows the dependence of the storage modulus at $\omega$ $=10 \mathrm{~s}^{-1}$ on the frequency of ac electric field with an amplitude of $8 \mathrm{kV} / \mathrm{mm}$, where dots and a line denote, respectively, the experimental data and the calculated result. The theoretical curve shifts to lower frequency. The origin of the discrepancy is not clear at present. It might be ascribed to the fact that the radius of the real column is dependent on the height; it becomes large near the plates.

\section{CONCLUSIONS}

A well-defined columnar structure was successfully constructed from a droplet-dispersed state subjected to an elec- tric field. The columnar structure was clearly observed with a CSLM. The storage shear modulus of the columnar structure was measured with changing the electric field. A linear relation was found between the storage shear modulus at low frequencies and the square of the electric field. The static modulus was calculated taking into account the Maxwell stress exerted on the interface and interfacial tension. The linear relation was well reproduced by the calculation without adjustable parameter. It was found that the low-frequency shear modulus depends on the frequency of the applied ac electric field; it decreases at high frequencies. This behavior was also explained qualitatively by the theory. In the present theory, only the static shear modulus was calculated. The dynamic shear modulus should be calculated in future.

\section{ACKNOWLEDGMENTS}

This work was partially supported by Grant-in-Aid for Scientific Research [Priority Area "Soft Matter Physics" (Contract No. 463), C (Contract No. 19540326), and Wakate B (Contract No. 19740255)] from the Ministry of Education, Culture, Sports, Science and Technology of Japan.
[1] K. Tajiri, K. Ohta, T. Nagaya, H. Orihara, Y. Ishibashi, M. Doi, and A. Inoue, J. Rheol. 41, 335 (1997).

[2] X. Pan and G. McKinley, J. Colloid Interface Sci. 195, 101 (1997).

[3] H. Kimura, K. Aikawa, Y. Masubuchi, J. Takimoto, K. Koyama, and K. Minagawa, Rheol. Acta 37, 54 (1998).

[4] H. Kimura, K. Aikawa, Y. Masubuchi, J. Takimoto, K. Koyama, and T. Uemura, J. Non-Newtonian Fluid Mech. 76, 199 (1998).

[5] H. Orihara, M. Doi, and Y. Ishibashi, Int. J. Mod. Phys. B 13, 1949 (1999).

[6] H. Orihara, Y. Hosoi, K. Tajiri, Y. Ishibashi, M. Doi, and A. Inoue, J. Rheol. 43, 125 (1999).

[7] J. Ha and S. Yang, J. Rheol. 44, 235 (2000).

[8] H. Orihara, T. Shibuya, T. Nagaya, and S. Ujiie, J. Phys. Soc. Jpn. 75, 063802 (2006).

[9] Y.-H. Na, T. Shibuya, S. Ujiie, T. Nagaya, and H. Orihara,
Phys. Rev. E 77, 041405 (2008).

[10] M. Trau, S. Sankaran, D. A. Saville, and I. A. Aksay, Nature (London) 374, 437 (1995).

[11] M. Trau, S. Sankaran, D. A. Saville, and I. A. Aksay, Langmuir 11, 4665 (1995).

[12] S. Y. Chou, L. Zhuang, and L. Guo, Appl. Phys. Lett. 75, 1004 (1999).

[13] E. Schaffer, T. Thurn-Albrecht, T. P. Russell, and U. Steiner, Nature (London) 403, 874 (2000).

[14] Z. Lin, T. Kerle, T. P. Russell, E. Schaffer, and U. Steiner, Macromolecules 35, 3971 (2002).

[15] S. Torza, R. G. Cox, and S. G. Mason, Philos. Trans. R. Soc. London, Ser. A 269, 295 (1971).

[16] A. Inoue and S. Maniwa, J. Appl. Polym. Sci. 55, 113 (1995).

[17] D. Graebling, R. Muller, and J. F. Palierne, Macromolecules 26, 320 (1993). 